\title{
Multi-Chip MODUles USING ETCHED GOLD CONDUCTORS AND A High PERFORMANCE LOW PERMITTIVITY PHOTOSENSITIVE DIELECTRIC
}

\author{
Gary Shorthouse, Allan Berzins, \\ Trevor Funnell \& John Smyth \\ Johnson Matthey Technology Centre \\ Sonning Common, England
}

As a response to the ever increasing demands made on advanced electronics interconnection, a new interconnection technology based on a combination of etched printed gold conductors and a new kind of inorganic dielectric has been developed. The gold conductors are patterned to provide dense interconnection networks with conductor line widths and spacings of less than $25 \mu \mathrm{m}(0.001 \mathrm{in})$. The dielectric forms insulating layers between these gold conductor planes and allows them to be interconnected through tiny holes (vias) in the dielectric of less than $50 \mu \mathrm{m}$ diameter, giving extremely high density three dimensional multi-layers. The dielectric also has a low permittivity (around 4) along with very low dielectric loss, necessary for high speed performance and reduction of cross-talk, and is hermetic. In addition,

relatively low costs are maintained. Since the first introduction of this system $[1,2]$ continual testing and improvements have been carried out and thousands of tiny vias have now been successfully incorporated in multi-layer circuitry. This paper reviews the latest results of this work, concentrating on both the processing and performance of the materials in high density multi-layer circuits. 


\section{INTRODUCTION}

The performance of modern high speed electronic systems is often dominated by the packaging and interconnection systems external to the integrated circuits. Optimum performance can only be achieved if the spacing between integrated circuits, electrical signal paths, parasitic capacitance and cross talk are minimized. This requirement has led to the development of the Multi-Chip Module (MCM), in which a number of unpackaged integrated circuits are interconnected on a high density single substrate. Alternative materials systems and processes have been developed for the manufacture of MCMs. Examples of these are laminated structures (MCM-L) which utilize advanced printed circuit board technology, co-fired ceramic structures (MCM-C), and polymer dielectrics with thin film conductors on ceramic and silicon substrates (MCM-D). Each of these technologies has associated advantages and problems, but in general terms both performance and cost increase in the order MCM-L, MCM-C, MCM-D with relative costs of approximately $1: 2: 3$. Numerous predictions of the potential future growth of $\mathrm{MCM}$ markets have been made with some estimates placing it as high as $\$ 20$ billion by the year 2000 .

This paper describes the development of a new MCM technology which can achieve circuit densities approaching those of MCM-D, yet with lower costs than MCM-C. Referred to below as the JM (Johnson Matthey) system, it is an advanced thick film system based on a combination of screen printed and etched gold conductors along with a new kind of low permittivity thick
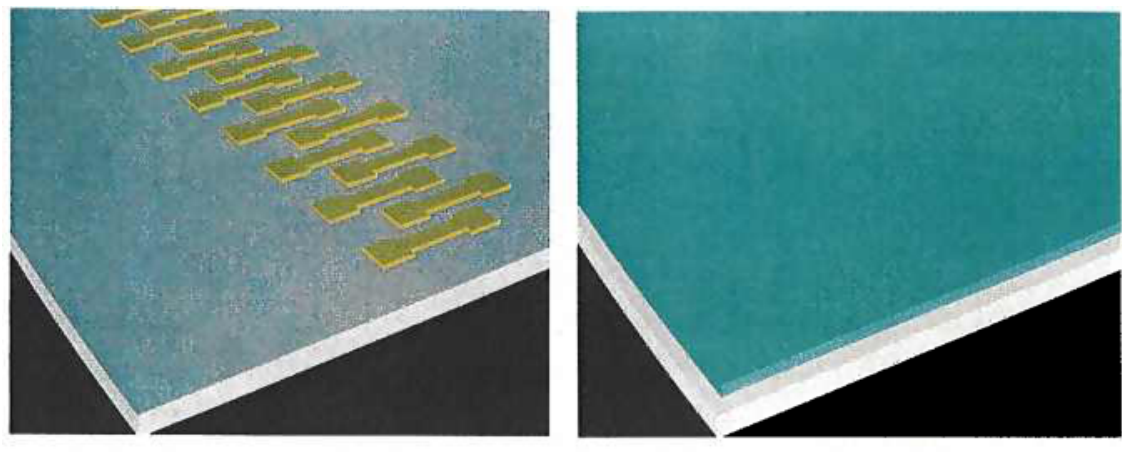

1. Substrate + gold one layer

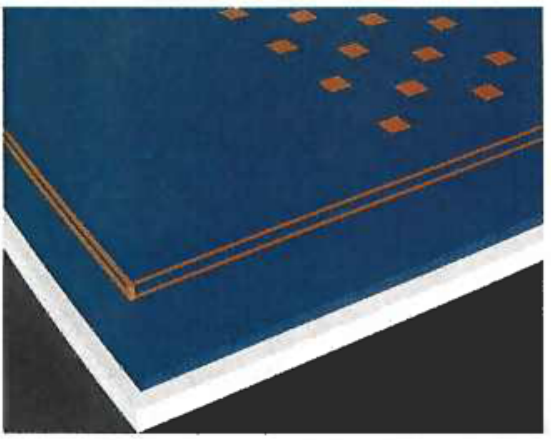

3. Exposure of dielectric through mask

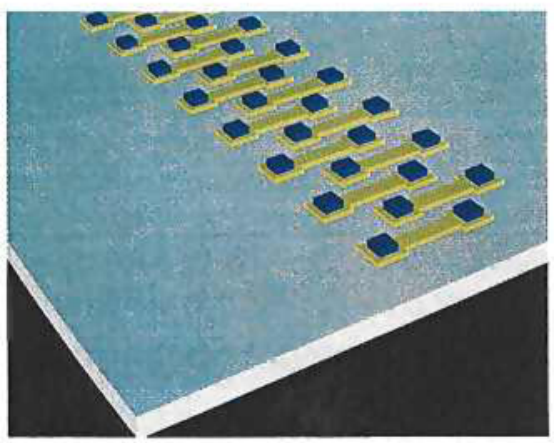

5. Skeletal view of gold one + vias in dielectric one

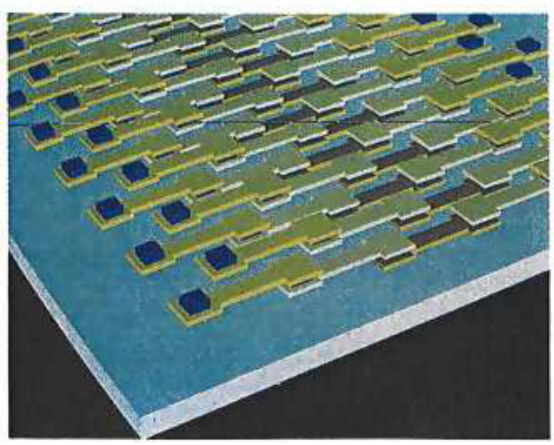

2. Screen Printed dielectric layer

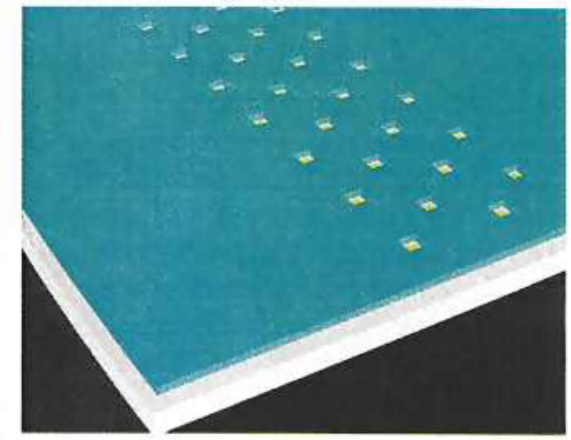

4. Vias developed trough dielectric layer

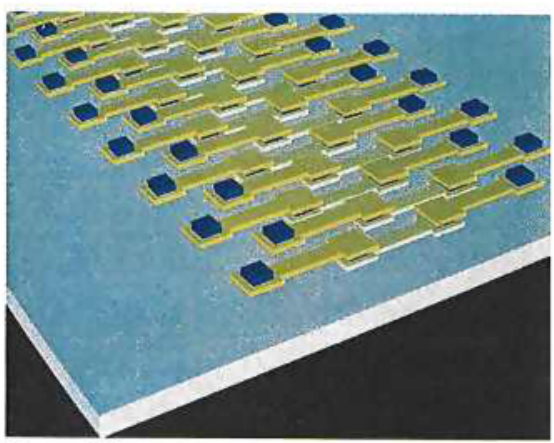

6. + gold two and vias in dielectric two

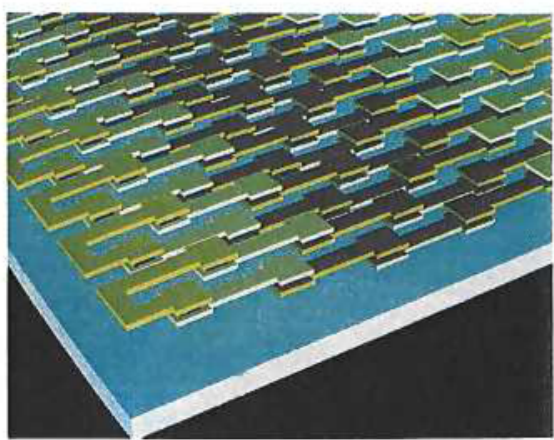

Figure 1

Processing steps involved in producing multi-chip modules 
film dielectric. The dielectric is formulated using novel inorganic powders that are produced from chemical precursors using sol-gel processing techniques. These are dispersed in a photosensitive vehicle to produce a screen-printable paste. Using a combination of screen printing and photo-processing, the dielectric can give via resolutions smaller than $50 \mu \mathrm{m}$. The fired films, which are purely inorganic, have a permittivity of around 4, very low dielectric loss and are fully dense.

\section{MATERIALS DEVELOPMENT}

\section{Gold Conductors}

For some time, gold conductor materials have been available that allow very dense conductor networks to be produced. Using both conventional [3] and specialist [4] screen technologies, in combination with special gold conductor thick film pastes $[5,6]$, lines and spaces down to $50 \mu \mathrm{m}$ can be printed. Etchable thick-film materials are also available which will permit lines and spaces down to $20 \mu \mathrm{m}$ to be produced using simple photoengraving, and metallo-organic gold materials can be printed, fired and etched to give line resolutions of $15 \mu \mathrm{m}$ or lower.

Until recently the benefits of these advanced conductor materials have only been realized in single layer applications such as thermal printer heads and the upper layer of multi-layer hybrids. This is due to the constraints imposed by the thilck film dielectrics that have thus far been available. These have suffered primarily from both poor via resolution and high dielectric constant. The smallest dielectric via sizes that can normally be achieved using screen printing techniques are around $250 \mu \mathrm{m}$. When compared with available conductor line resolutions of less than 20 $\mu \mathrm{m}$, it is apparent that such relatively large vias severely limit overall circuit density.

The permittivity of conventional thick film dielectrics is too high for good high frequency performance and considerably lower permittivities were required. The advantage of a low permittivity dielectric is that the capacitance between the conductors is reduced, dielectric losses are minimizzed, reduced levels of cross-talk are achieved and signal rise and decay times are improved.
Thus, in order to utilize the full potential of the advanced gold conductors in high speed multi-layer circuitry, it was necessary to develop a completely new type of dielectric.

\section{Thick Film Dielectrics}

Most conventional thick film dielectrics are based on low melting point lead-containing glasses along with various fillers (e.g. alumina) which are used to both modify the thermal expansion coefficient of the fired film and to impart re-fire stability. The high atomic number elements present in these materials are responsible for their relatively high dielectric permittivities, typically 9 to 12 . A number of authors have described various strategies for reducing the permitrivity of thick film dielectrics, including the use of glass ceramic systems and the deliberate incorporation of air (porosity) within the dielectric layers. Examples of the latter are the use of hollow silica micro-spheres [7], and reactive dielectrics which swell during firing to produce air bubbles in the films [8]. Whilst these techniques have produced films with low permittivities, the use of non-homogeneous materials can lead to dispersion loss at high frequencies, process sensitivity can be a significant problem and the materials do not have the ability to form small vias. In order to circumvent these problems a fully dense low permittivity dielectric material was required that could provide vias with sizes comparable to the available conductor geometries previously described.

\section{Photosensitive Sol-Gel Derived Dielectric}

The dielectric that underpins this development consists of inorganic powders manufactured using sol-gel technology in combination with a photosensitive vehicle. The powders are produced by the hydrolysis and condensation of various metal alkoxides (Figure 2) to

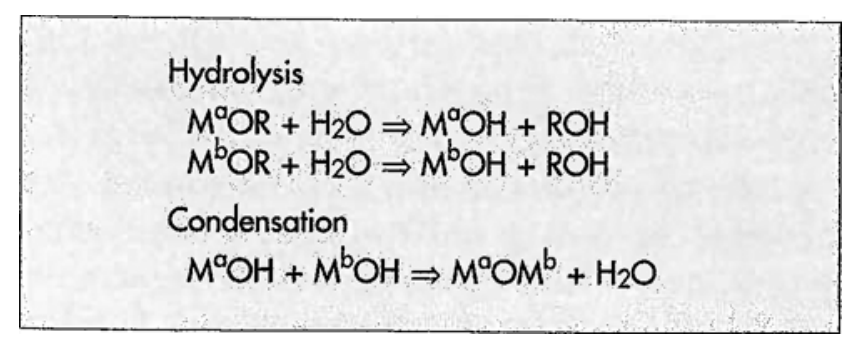

Figure 2

The Sol-Gel Process 


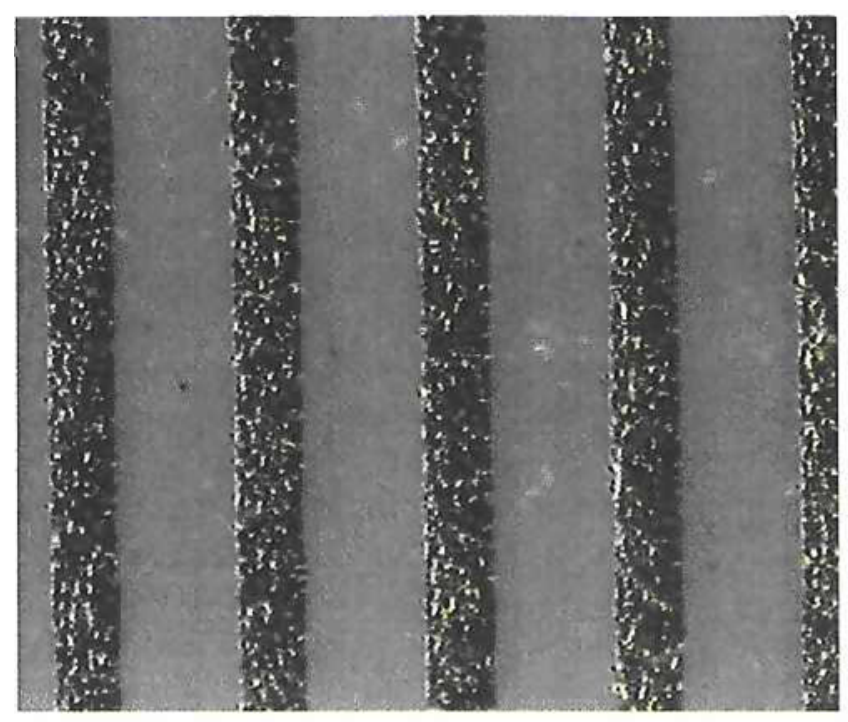

Figure 3

$50 \mu \mathrm{m}$ gold conductor tracks

form a polymeric structure that, depending on reaction conditions, is either precipitated as a solid or forms a gel from which the solvent is subsequently removed. After additional treatments, highly reactive powders are formed which will sinter to give fully dense films at temperatures hundreds of degrees lower than conventionally produced materials of the same composition. The highly reactive nature of the powder is associated with a high degree of internal micro-porosity trapped within the powder. The micro-porosity, which collapses during sintering, imparts considerable surface energy.

This technique has allowed us to use low permittivity materials that would normally have sintering temperatures considerably above the maximum for use with gold conductors. Additionally, sintering is achieved without the addition of the usual alkali metal fluxing components necessary in other thick film dielectrics. The removal of these mobile constituents in combination with the use of very pure starting materials has enabled us to achieve unprecedentedly low levels of dielectric loss.

Mixtures of powders with different chemical compositions are used in order to achieve the required properties. These are the ability to sinter to give dense crack and pinhole-free layers, refire stability, excellent electrical properties, hermeticity and their ability, in combination, with a photosensitive vehicle, to provide tiny vias and maintain them during firing.

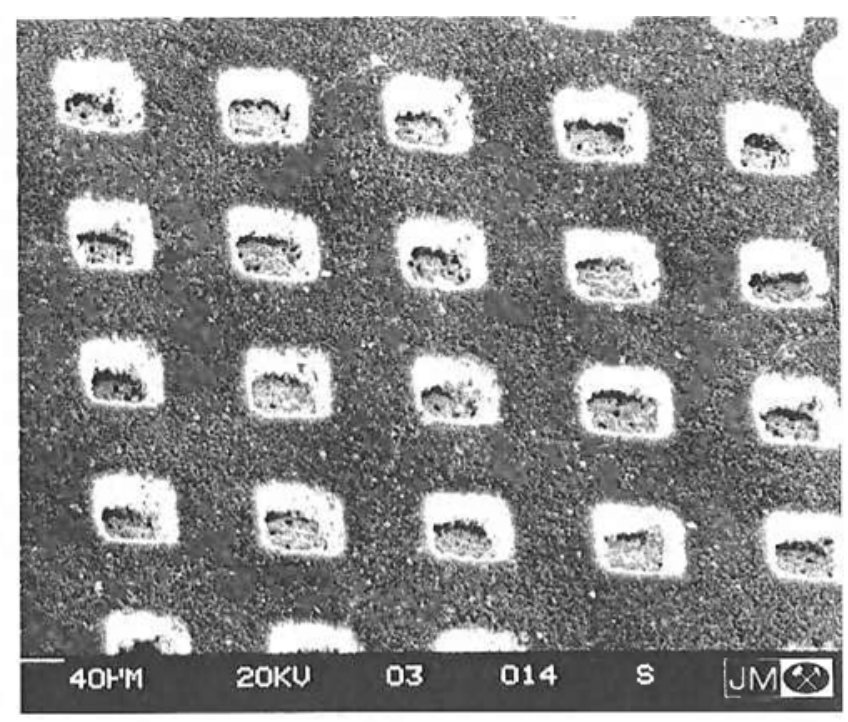

Figure 4

$50 \mu \mathrm{m}$ gold unfired via array

The photosensitive vehicle in which the powders are dispersed is composed of an ultraviolet curable monomer, a photoinitiator, thermoplastic materials, solvents, surfactants, and rheology modifying additives. These are completely removed during the firing process to leave no detectable residue.

\section{PROCESSING}

The processing steps for producing a multi-layer circuit are shown schematically in Figure 1 (page 128). The individual steps are described below.

\section{Gold Layers}

Initially the gold conductor paste is screen printed on alumina and fired to give a 4 to $5 \mu \mathrm{m}$ thick layer. This can be carried out as a single operation, however for maximum density, two or three thin gold layers may be printed individually with either a single or separate firings at 850 to $950^{\circ} \mathrm{C}$. The gold is then photoengraved using a conventional positive photoresist process and is etched in iodine/potassium iodide. Etching may be carried out by either dipping in hot etchant or using a spray etcher such as commonly used for printed circuit board etching. Figure 3 shows $50 \mu \mathrm{m}$ tracks produced in this manner. When the gold is printed on dielectric, due to the extremely high adhesion at the gold-dielectric interface, improved etching is achieved 


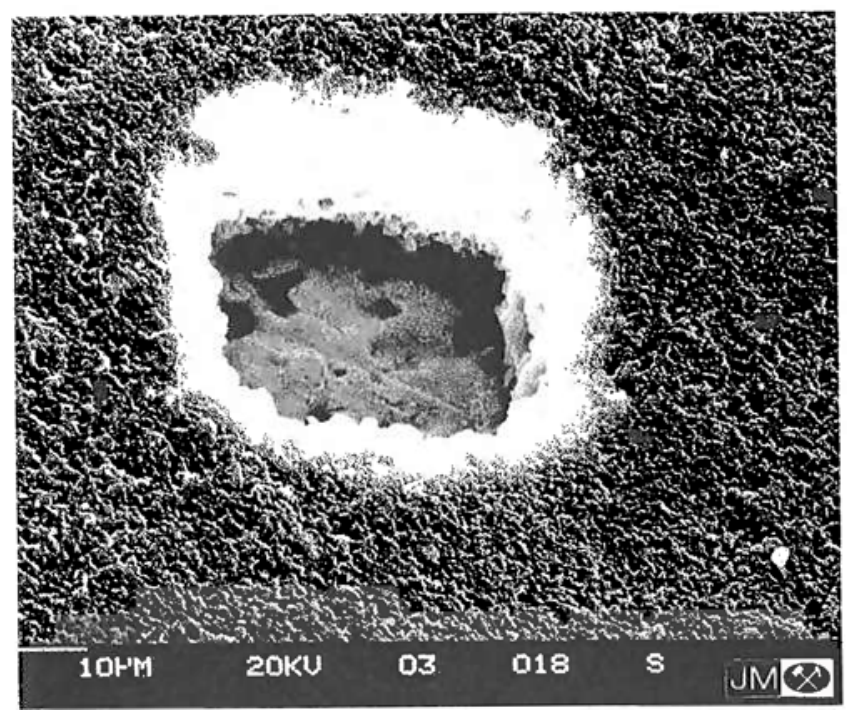

Figure 5

Unfired single via

if the gold is pre-fired at $750{ }^{\circ} \mathrm{C}$, photoengraved, and post-fired at 850 to $950^{\circ} \mathrm{C}$.

\section{Dielectric Layers}

The dielectric is screen printed, allowed to level, aligned with a via photo-mask and exposed to ultraviolet light which causes the vehicle within the exposed areas to polymerize and bind the inorganic powders. Exposure times are dependent on the equipment used, but times of around 5 seconds would be typical. It is

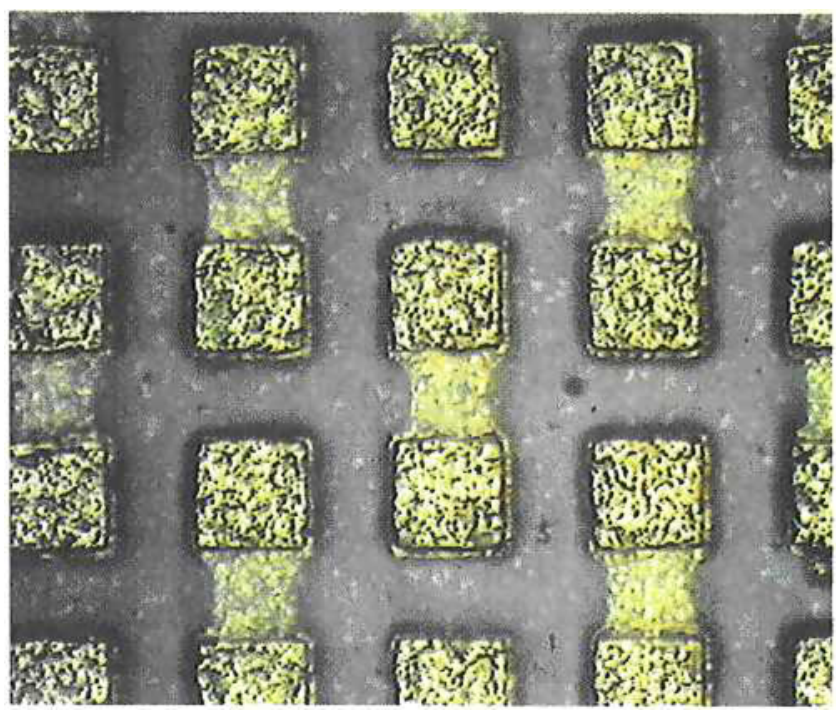

Figure 7

Double stacked filled vias

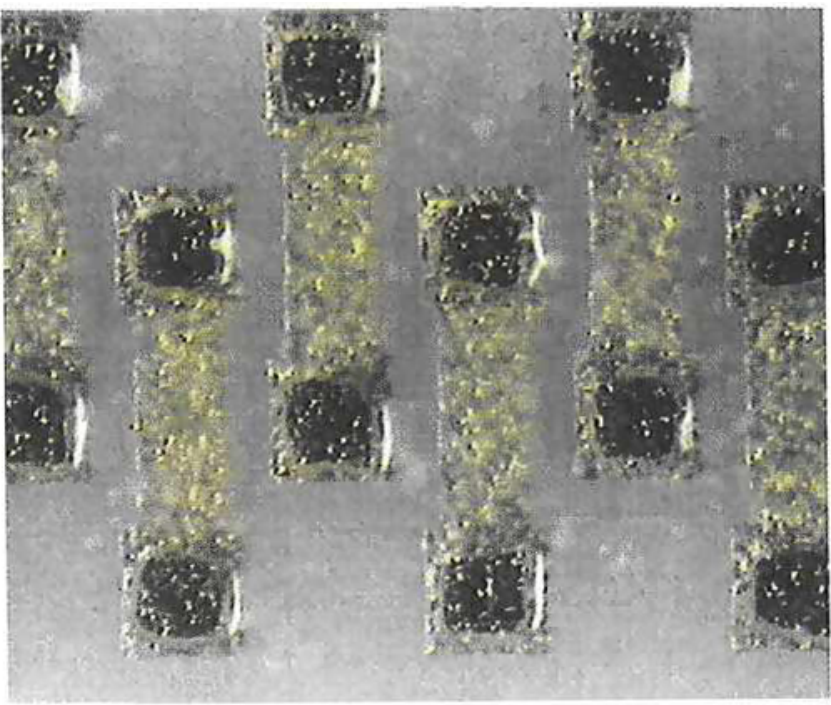

Figure 6

Fired vias over gold conductors

then spray developed with isopropyl alcohol using a hand spray or automatic spray development unit. Figure 4 shows an array of vias produced using a $50 \mu \mathrm{m}$ mask at this stage in the process and Figure 5 a single via at higher magnification.

Following development, the dielectric is dried at around $140^{\circ} \mathrm{C}$, and then fired at around $900^{\circ} \mathrm{C}$. During firing, the organic vehicle is completely removed and the powder sinters to form a dense glassy layer whilst leaving the vias open. Figure 6 shows fired vias in the dielectric over gold conductors. Note that the fired dielectric is transparent and so the underlying gold is visible.

\section{Via Filling}

The via walls are smoothly tapered at an angle of approximately $40^{\circ}$ and this allows them to be readily filled with conductor paste during a simple screen printing operation. For single dielectric layers this occurs automatically during the printing of the next gold conductor layer, however a separate via filling operation may be necessary if multiple dielectric layers are used between the conductor planes. This is carried out by screen printing the regions of the dielectric containing vias with gold paste, firing (preferably at $750^{\circ} \mathrm{C}$ ) and then photoengraving to remove the unwanted gold as described above. Figure 7 shows vias in double dielectric layers that have been filled in this manner. 


\section{Subsequent Processing}

The above process steps are repeated sequentially to build up the multi-layer structure. Figure 8 shows a circuit after adding the top gold layer. Test circuits containing four conductor layers have been constructed and thousands of vias down to $50 \mu \mathrm{m}$ size have been satisfactorily interconnected with via densities of around 400 per $\mathrm{cm}^{2}$. The dielectric also gives a high degree of planarisation, enhancing the multilayer capability of the system.

\section{PERformanCE AND COST}

\section{Properties and Comparative Performance}

The major properties of the system along with those of conventional thick film technology are listed in Table 1, and the overall performance of the system is compared with thick film and other MCM technologies in Figure 9.

It is apparent that the JM system offers a major improvement on thick film technology in all aspects of performance. The permittivity of the dielectric is comparable to that of MCM-L and MCM-D technologies and represents a considerable improvement over thick film and MCM-C, making it highly suitable for high speed circuitry and low levels

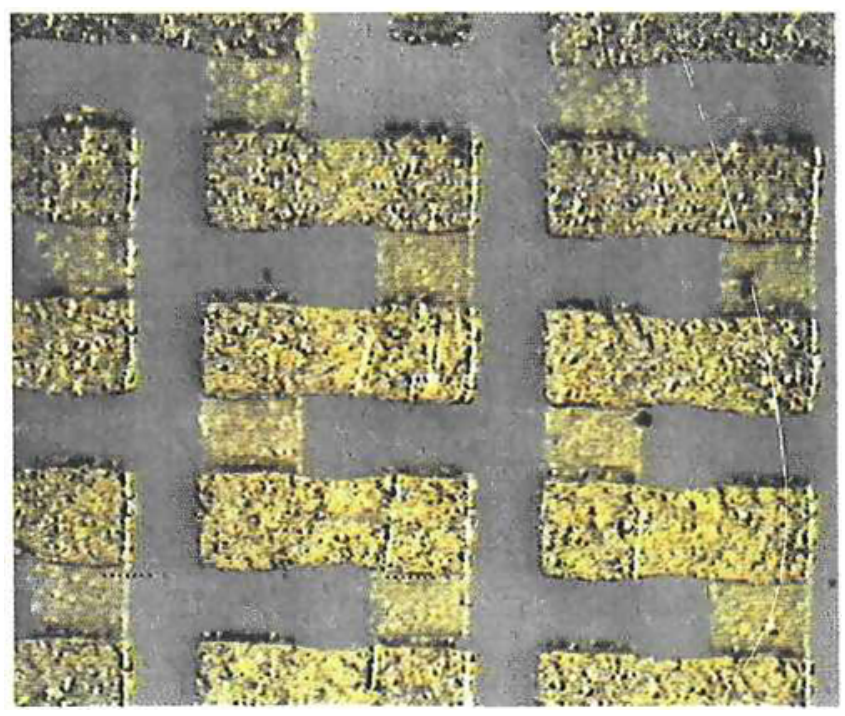

Figure 8

Vias interconnected through two gold layers
Table 1

Properties

\begin{tabular}{|c|c|c|}
\hline PROPERTY & $\begin{array}{c}\text { NORMAL } \\
\text { THICK FILM }\end{array}$ & JM SYSTEM \\
\hline $\begin{array}{c}\text { Conductor } \\
\text { geometry }\end{array}$ & $\begin{array}{c}125 \mu \mathrm{m} \\
\text { line } / \text { space }\end{array}$ & $\begin{array}{c}25 \mu \mathrm{m} \\
\text { line } / \text { space }\end{array}$ \\
$\begin{array}{c}\text { Conductor } \\
\text { thickness }\end{array}$ & $10 \mu \mathrm{m}$ & 3 to $5 \mu \mathrm{m}$ \\
$\begin{array}{c}\text { Dielectric } \\
\text { thickness }\end{array}$ & $50 \mu \mathrm{m}$ & $20 \mu \mathrm{m}$ \\
$\begin{array}{c}\text { Via resolution } \\
\text { Permittivity }\end{array}$ & $\begin{array}{c}250 \text { to } \\
8 \text { to } 14\end{array}$ & 25 to $50 \mu \mathrm{m}$ \\
$\begin{array}{c}\text { Dielectric loss } \\
\text { Leakage }\end{array}$ & 0.001 to 0.003 & 3.8 to 4.2 \\
current, $\mu \mathrm{A}$ & 1 to 20 & $<5.10^{-5}$ \\
& & $<5.10^{-5}$ \\
\hline
\end{tabular}

of cross-talk. The dielectric loss is the lowest of all the alternative technologies. Due to the a combination of small via size and fine conductor widths, the interconnection density per layer approaches that of MCM-D, permitting a large degree of circuit miniaturization. The leakage current under bias in a saline electrolyte is three orders of magnitude less than that of thick film dielectrics, which indicates a highly hermetic dielectric [9].

The gold conductor may also be reliably wirebonded using either gold or aluminium wire.

\section{Costs}

Although this technology is based on gold conductors whilst some of the alternative systems use lower cost palladium/silver or copper conductors, the total substrate system cost is still relatively low. This is due to a number of factors: the quantity of gold used is minimized, as the conductor thickness may be only 3 or $4 \mu \mathrm{m}$ and the high degree of miniaturization offered by the technology means that the total length of conductor lines is kept to a minimum. In MCM$\mathrm{C}$ co-fired technology many circuit layers, perhaps 20 to 40 , may be required in order to achieve the required circuit functionality, however, the fine feature sizes available in the JM technology can reduce this 

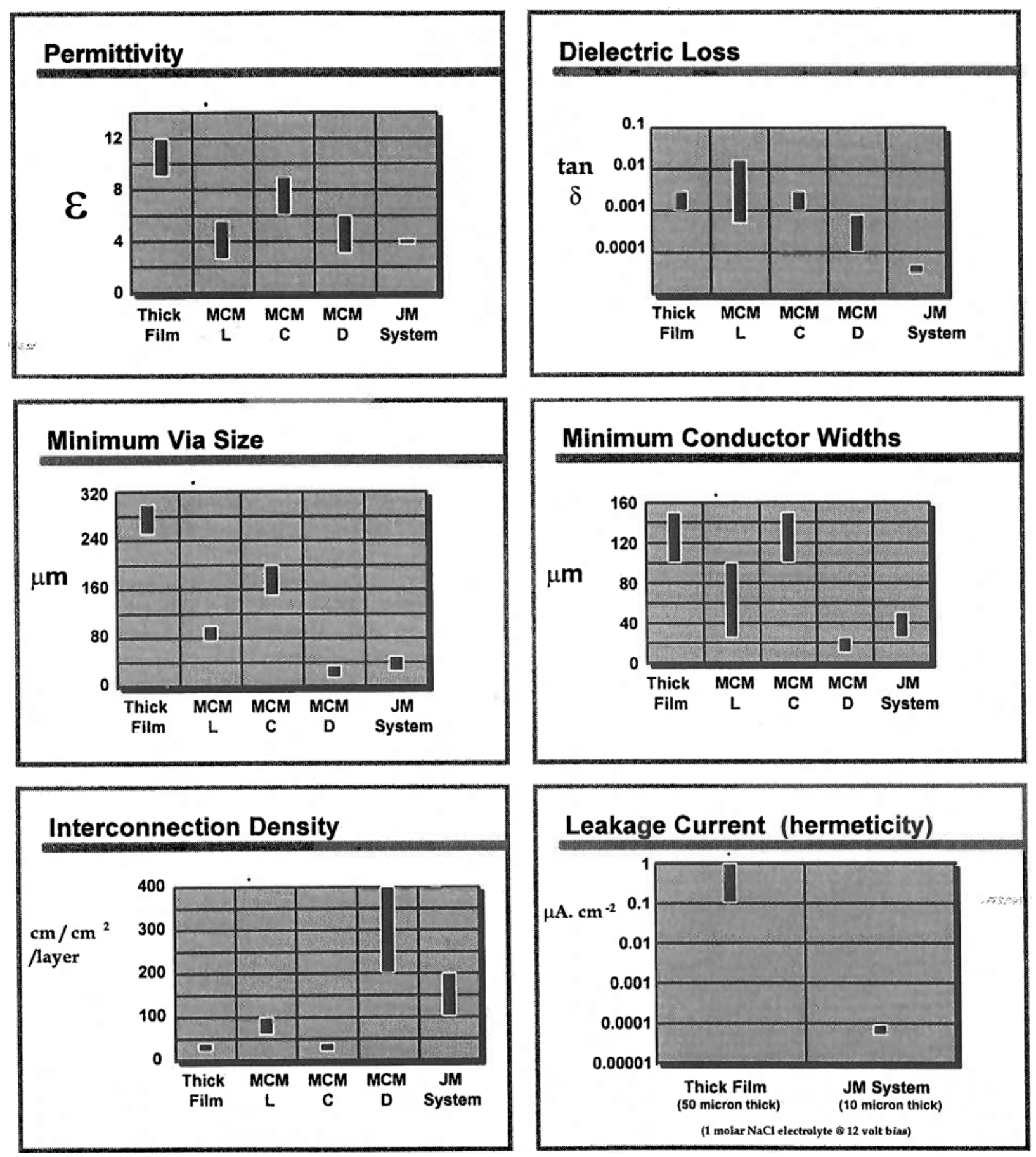

Figure 9

Comparative performance

to perhaps 4 to 7 layers, with considerable savings in both conductor and dielectric materials. Whilst technologies such as MCM-C and MCM-D require substantial investment in plant and processes, the JM system is based on low-cost thick film processes and although some additional photo-processing stages are necessary, relatively small additional capital investment is required. Taking these and additional factors into account, the overall cost of the JM system lies between that of MCM-L and MCM-C. 


\section{OVERVIEW}

A new high density interconnection technology for MCMs and high speed multi-layer hybrids based on gold conductors and a novel inorganic dielectric has been developed. The system achieves performance and circuit density approaching that of MCM-D whilst maintaining relatively low costs.

\section{REFERENCES}

1. G. Shorthouse, L. Bricknell, R. Russell, R. Morris, 'A low permittivity high resolution dielectric for high performance thick film interconnections', Proc. High Density Interconnection Seminar, ISHM, U.K. October 1990

2. G. Shorthouse, L. Bricknell, R. Russell, R. Morris, 'High density, hỉgh speed thick film interconnections incorporating a new low permittivity high resolution dielectric', Proc. International Sym-posium on Microelectronics, Chicago, October 1990
3. B. Carr, 'An Introduction to Thick Film Fine Line Circuitry', Proc. 8th European Hybrid Microelectronics Conference, Rotterdam, 1991

4. G. Shorthouse, B. Walton, 'Precision Printing for High Density Interconnections', Proc. 6th European Hybrid Microelectronics Conference, Bournmouth, 1987

5. D. Savage et al., 'Ultra fine line thick film golds using photolithographic techniques', Hybrid Circuit Technology (Sept 1989)

6. T. Stewart, Y. Zeniya, 'Advanced Gold conductor compositions for hybrid circuit applications', Proc. 6th European Microelectronics Conference (1987)

7. D. Kellerman, 'The Development and characterization of a low dielectric constant thick film material', Proc. of 7 th Electronic Components Conference, Boston, (1987)

8. A. Shaikh, D. Hankey, K. Allison, 'New Materials for High Speed Multilayer Circuits', Proc. 8th European Hybrid Microelectronics Conference, Rotterdam, 1991

9. C. Needes, D. Button, "Reliability Testing of Thick Film Multilayer Materials', ECC Proc., Washington DC, 1985 pp 505-511 